\title{
Necesidad de la revisión de los estudios de amenaza sísmica a raíz del sismo de Tohoku de 2011
}

\section{Need for review of seismic threat studies due to Tohoku ear thquake 2011}

Maryorie Correa Leguizamón

Tecnóloga en Construcciones Civiles. Investigadora de la Universidad Distrital Francisco José de Caldas. Bogotá, Colombia.mcorreal@correo.udistrital.edu.co

\section{Andrés José Alfaro Castillo}

Ingeniero civil, magíster en Ingeniería Sísmica y Dinámica Estructural. Ingeniero de Ingetec S.A. Bogotá, Colombia.andresalfaro@ingetec.com.co

Clasificación del artículo: Investigación (Conciencias)

Palabras clave: Amenaza, sismo, Tohoku.

Key words: Threat, Earthquake, Tohoku.

\section{RESUMEN}

Este artículo plantea la necesidad de la revisión y actualización de los estudios de amenaza sísmica en Colombia, a raíz de los catastróficos efectos del sismo de Tohoku del 11 de marzo de 2011 en Japón en razón a: a) la insuficiente instrumentación sismológica en Colombia; b) la insuficiente información de las fallas geológicas activas; c) la inexistente instrumentación en el área de los tsunamis, a pesar de haber sufrido varios en el siglo XX, en especial el de magnitud $\mathrm{Mw}=8.8 \mathrm{del}$ 31 de enero de 1906; d) la norma colombiana de construcción sismorresistente no es conservadora; e) resultados de investigaciones muestran valores de pga mayores a la norma vigente.

\section{ABSTRACT}

This paper discusses the need for reviewing and updating the seismic hazard studies in Colombia following the catastrophic effects of the Tohoku earthquake March 11th, 2011 in Japan, knowing that: a) Insufficient seismological instrumentation Colombia; b) Insufficient information on active fault lines; c) Lack of implementation in the area 


\section{con-ciencias}

of the tsunami, in spite of having suffered several in the twentieth century, especially the magnitude $\mathrm{Mw}=8.8$ of January 31,1906 ; d) The Colom- bian earthquake-resistant construction standard is not conservative; e) Results of research show pga values greater than the current standard.

\section{INTRODUCCIÓN}

Japón es talvez el país más desarrollado en los temas de prevención y mitigación de desastres de tipo sísmico y tsunamis. Tiene análisis de eventos sísmicos que se remontan al año 599, han sido pioneros en instrumentación, análisis de la información y la posterior implementación de medidas, tanto a nivel de las construcciones, como del comportamiento de las personas ante una crisis sísmica.

Sin embargo, los estimativos de acción sísmica y altura de las olas del tsunami se quedaron cortos con respecto a los eventos precursores, el mayor con Mw de 7.2; el megasismo del 11 de marzo de 2011, con magnitud Mw de 9.0 y el posterior tsunami con altura de ola de 7.6 metros en la estación de lioka y réplicas con magnitudes $\mathrm{Mw}$ entre $6,1 \mathrm{y}$ 7,2; que adicional a la lamentable pérdida de vidas, los desaparecidos, los heridos y los damnificados, ha generado una crisis nuclear sin precedentes a nivel mundial, con fallas importantes no sólo en la planta de Fukushima, sino en otras plantas, tanto al norte como al sur de la zona epicentral (Earthquake Research Institute, 2011) [1].

El evento del 11 de marzo de 2011 se ha considerado como el peor evento en los últimos 140 años en Japón. Las aceleraciones pico del suelo han superado las previsiones, este evento generará una revisión de los estudios de amenaza sísmica en Japón y también en el resto del mundo.

En Colombia se han realizado múltiples estudios de amenaza sísmica, tanto a nivel nacional como en ciudades específicas y para proyectos grandes, entre los cuales se destacan los proyectos hidroeléctricos. De alguna manera, los estudios nacionales no han sido conservadores, han sido, tal vez, demasiado complacientes con los constructores, con el fin de no incrementar los costos de construcción, a pesar de que dicho incremento es bastante marginal.

Estudios realizados independientemente a nivel académico y para proyectos especiales como hidroeléctricas han mostrado valores de aceleración pico del suelo superiores a los estudios nacionales, de alguna manera "oficiales", sin embargo, los estudios nacionales han sido incorporados a la Norma Colombiana de Construcción Sismoresistente.

A nivel de ejemplificación, en este artículo se presenta la evaluación de la amenaza sísmica regional para la ciudad de Barrancabermeja, utilizando el estado del arte en la evaluación probabilista y determinista en la cuantificación de la amenaza. Los resultados obtenidos son entre 190 y $235 \%$ mayores que los valores de la norma vigente NSR-10, para una vida útil de las estructuras de 50 años y probabilidades de excedencia del 10\%.

La lección de Sendai (Japón) del 11 de marzo de 2011 implica la necesidad de la revisión y actualización de los estudios de amenaza sísmica. Barrancabermeja es una ciudad colombiana ubicada en el departamento de Santander, es sede de la refinería de petróleo más grande de Colombia, con una población aproximada de 300.000 habitantes. Esta ciudad se encuentra localizada en una zona que presenta una amenaza sísmica importante a causa del ambiente tectónico regional, dentro de las fuentes sismogénicas de mayor efecto se encuentran las fallas Bagre, Otú, Infantes, Nus, 


\section{con-ciencias}

Bucaramanga-Santa Marta, Palestina, Cimitarra, Arrugas, La Salina, El Carmen y la zona del Nido de Bucaramanga.

\section{ESTADO DEL ARTE}

Existen estudios geológicos realizados por el Ministerio de Minas y Petróleos en 1966 [2], que comprenden la geología del cuadrángulo H-11. En la parte sísmica se cuenta con el estudio de reconocimiento de microsismos en Bucaramanga, que fue desarrollado dentro del proyecto cooperativo "Nariño II" por el Instituto Geofísico de los Andes Colombianos con la colaboración de la Universidad de Wisconsin, Madison y de la Universidad del Valle, en 1981 (Goberna, 1981) [3].

También en la parte sísmica se encuentra el estudio de Geología Sísmica y Sismicidad del Noroeste de Colombia, desarrollado por Page (1986) [4], el cual contiene abundante información sobre el marco tectónico de la región de Santander y fallas activas. En cuanto a estudios específicos para la ciudad de Barrancabermeja, se tiene el de Flórez (1993) [5].

De igual forma es importante la información contenida en el estudio General de Amenaza Sísmica para Colombia (AIS et ál, 1996) [6]. En el año 2000 el Instituto Geofísico Universidad Javeriana y Consultoría Colombiana realizaron la Microzonificación Sísmica Preliminar de la Ciudad [7], [8]; posteriormente, González-Rodríguez (2001) [9] realizó un estudio de riesgo teniendo en cuenta la ubicación de desplazados en zonas vulnerables (González-Rodríguez y Alfaro, 2001) [10]. Este artículo presenta algunos de los hallazgos más importantes de Correa (2011) [11].

\section{METODOLOGÍA}

Esta investigación utiliza datos geológicos y sismológicos para la evaluación de la amenaza sís- mica. Por tanto se recopiló la información referente a las estructuras tectónicas; por otra parte se recopiló y analizó la información de sismos ocurridos en la región. Con la información de las fallas geológicas se estimó el sismo máximo creíble; con la información sismológica se generaron las curvas de amenaza sísmica. A continuación se presenta cada aspecto en forma detallada.

Un estudio de amenaza sísmica debe identificar los diferentes componentes de la amenaza sísmica del área de estudio. El estudio debe, por tanto, incluir las estructuras tectónicas activas. Los métodos utilizados para lograr este objetivo varían en complejidad dependiendo del grado de precisión deseado, el cual depende del nivel de amenaza de la zona de interés y de los recursos disponibles. El nivel de estudio más simple, se basa generalmente, en la recopilación e interpretación de la información disponible, es el menos costoso de los estudios.

Un estudio de amenaza sísmica debe identificar las estructuras tectónicas activas de la zona y determinar las características de las fallas. La caracterización deberá establecerse, a partir de la información existente, en la que estructuras actuales hayan sido detectadas previamente. Para ello cabe identificar el tipo de falla: normal, inversa, o de deslizamiento lateral; también su longitud, azimut, inclinación, tasa de movimiento, y sismos asociados.

Es necesario identificar las fallas a partir de las fuentes bibliográficas consultadas e indicar aquellas en que se sospeche actividad reciente o actual.

La acción sísmica sobre una estructura se expresa generalmente como una función de una aceleración nominal, que es el resultado del estudio de amenaza sísmica regional; de las características de la estructura y de los factores que dependen de los efectos locales. En este caso los resultados son aceleración pico del terreno en roca. 


\section{con-ciencias}

\subsection{Evaluación probabilística de la amenaza sísmica para Barrancabermeja}

La zona de estudio comprende la región acotada entre las siguientes coordenadas geográficas: latitudes entre $8.069^{\circ} \mathrm{N}$ y $9.069^{\circ} \mathrm{N}$ y longitudes entre $72.86^{\circ} \mathrm{W}$ y $74.86^{\circ} \mathrm{W}[11]$.

El primer paso consistió en hacer una revisión de eventos sísmicos ocurridos en el área, para esto se realizó un proceso de recopilación de información de interés, teniendo en cuenta diversos catálogos y registros de los eventos sísmicos sentidos en Colombia (Correa, 2011) [11].

La información sobre sismicidad, se basa en los catálogos del proyecto SISRA, Programa para la Mitigación de Efectos de los Terremotos en la Región Andina, que comprende sismos entre los años de 1471 y 1981, y los catálogos del PDE (Preliminary Determination of Epicenters) para los sismos ocurridos entre 1981 y 2010 [12].

Al unificar las magnitudes y realizar el conteo de los sismos ocurridos con $\mathrm{Ms} \geq 4$, se encuentra el registro de 174 sismos en Barrancabermeja a partir de año 1700 . Pero de acuerdo con el proceso estacionario de Poisson de ocurrencia de sismos en el tiempo, la ventana de tiempo que se ajusta a este proceso es desde 1955 hasta 2011, con 162 sismos de magnitud Ms $\geq 4.0$.

La evaluación de la amenaza sísmica involucra la estimación del movimiento del suelo que será producido por futuros sismos. Esto se logra a través del uso de relaciones de atenuación que predicen valores de parámetros seleccionados del movimiento del suelo, en este caso de la aceleración, como una función de otros parámetros sísmicos tales como la magnitud y la distancia de la fuente sísmica al sitio.

Varias ecuaciones han sido propuestas para determinar la atenuación del movimiento del sue-

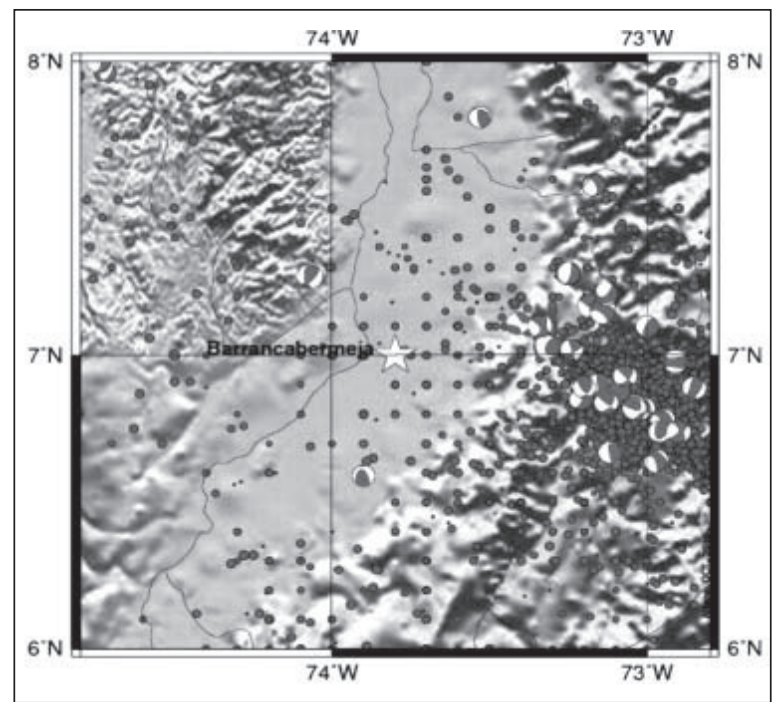

Fig. 1. Epicentros y mecanismos focales de Barrancabermeja (Correa, 2011) [11].

lo. Para este caso se tomaron tres ecuaciones, seleccionadas porque la magnitud se expresa en Ms o Mw, porque responden a sismos por fallas geológicas, porque son actuales y robustas. Las ecuaciones fueron: Patwardhan, et ál (1978) [13]; Fukushima \& Tanaka (1990) [14] y Si \& Midorikawa (1999, 2000) [15], [16].

\subsection{Estimación determinista de la amenaza sísmica Barrancabermeja}

Para la evaluación determinística se realiza una estimación de la posible magnitud $(\mathrm{Mw})$ que se presentaría en las fallas geológicas relativamente cercanas a la ciudad de Barrancabermeja. Esto es posible mediante la utilización de la ecuación planteada por Wells y Coppersmith (1994) [17].

\subsubsection{Estructuras tectónicas para Barrancabermeja}

Barrancabermeja se encuentra ubicada cerca de una de las zonas de mayor actividad sísmica de Colombia, en lo que se ha denominado el "nido" de Bucaramanga, donde se presentan una gran 


\section{con-ciencias}

cantidad de sismos agrupados en un área relativamente pequeña.

A continuación se describen las fallas geológicas por las que se vería afectada la ciudad, la información ha sido extractada del Instituto Geofísico Universidad Javeriana y Concol $[7-9,18,19]$.

\section{- Falla Bagre}

Es un ramal occidental de la Falla Palestina (Page, 1986) [4], es una falla de rumbo, con movimiento lateral izquierdo e inversa, con una longitud de $159.3 \mathrm{~km}$, orientada según rumbo NS, probablemente inclinada con ángulo alto hacia el oriente.

\section{- Falla El Carmen}

La falla El Carmen marca el límite más occidental de las unidades sedimentarias del Mioceno. Se calcula que cerca a la superficie los planos de falla tienen una inclinación cercana a los $30^{\circ}$, convergencia oriental. No se tiene registro de actividad en el Holoceno y hacia los ríos Guineo y Mocoa las estructuras están fosilizadas por los depósitos sedimentarios del cuaternario reciente. Tiene una longitud de $62 \mathrm{~km}[18,19]$.

\section{- Falla Santa Marta - Bucaramanga}

Santa Marta - Bucaramanga es un sistema de fallas importantes que se extiende por la costa del Caribe de la Cordillera Oriental a la medida de $6.5^{\circ} \mathrm{N}$, al sur de la ciudad de Bucaramanga. Tiene una Longitud de: $537,6 \mathrm{~km}$, rumbo de: $-19.1^{\circ} \pm$ 23; es una falla importante con movimiento sinistral (lateral izquierdo), y un desplazamiento de unos $110 \mathrm{~km}[18,19]$.

\section{- Falla Otú Norte}

La Falla Otú Norte discurre en dirección SurSureste y aparece como un desplazamiento de la Falla La Palestina. Se extiende en la dirección noroeste cerca del Bajo Nechí, donde está cubierta por depósitos recientes del Cuaternario. El extremo norte aparentemente se disgrega en varias fallas que cruzan el río Nechí. La falla tiene una longitud de $144.4 \mathrm{~km}$, un rumbo promedio de $-13.4^{\circ} \pm 8^{\circ}$, es inversa con movimiento siniestro lateral. Se asume que su comportamiento general es similar a su falla vecina La Palestina $[18,19]$.

\section{- Falla La Palestina}

La falla de La Palestina se extiende desde el departamento de Antioquia en el norte hasta la zona del volcán-nevado del Ruiz al sur. Se extiende a lo largo de la ladera oriental de la Cordillera Central de Colombia, desplazando rocas cristalinas metamórficas del Paleozoico, y en menor medida rocas plutónicas del Mesozoico. Las rocas están principalmente en el bloque occidental, las cuales elevan una probable superficie de erosión del Mioceno cuyos materiales remanentes se caracterizan por estar alineados en bancas estrechas y planas.

La geometría de la falla es la siguiente: longitud acumulada de $430.6 \mathrm{~km}$, rumbo promedio de $17.8^{\circ} \pm 11^{\circ}$, el sentido del movimiento es inverso siniestro lateral. Generalmente el lado occidental está levantado, sin embargo, también en la falla inversa se presenta el lado oriental hacia arriba.

\section{- Falla Cimitarra}

La falla de Cimitarra es una ramificación de la falla de La Palestina tomando una dirección nororiental en el borde oriental de la Cordillera Central. La falla desplaza rocas volcánicas del Cretáceo, rocas ígneas del Mesozoico, superficies erosionadas del Terciario en la Cordillera Central y sedimentos tardíos del Cuaternario. Partes de la falla son de la época del pre-plioceno debido a que están localmente cubiertos por sedimentos no deformados del Plioceno. En la 
parte nororiental la falla está cubierta por depósitos aluviales jóvenes de la parte media del valle del Magdalena.

La geometría de la falla es la siguiente: longitud $136.7 \mathrm{~km}$, rumbo promedio de $37.0^{\circ} \pm 3^{\circ}$, el sentido del movimiento es inverso siniestro lateral. La falla está marcada por escarpes bien conservados, trazas rectas largas, drenajes desplazados los cuales forman cursos de ríos bien alineados [18].

\section{- Falla Suárez}

Se extiende a lo largo de los Ríos Oro y Suárez, hasta interceptar la falla de Santa Marta-Bucaramanga a pocos kilómetros al norte de la ciudad. La falla desplaza verticalmente una superficie de erosión Terciaria más o menos $600 \mathrm{~m}$ y afecta los depósitos aluviales de la Meseta de Bucaramanga. Julivert $(1961,1963)$ [20-22] fue el primero en reconocer la actividad Cuaternaria de esta fa1la. En gran parte de su trayectoria coloca en contacto rocas Jurásicas sobre rocas Cretáceas. Al occidente de la ciudad sobre la margen izquierda del Río Oro, pone en contacto rocas sedimentarias de la formación Girón y depósitos cuaternarios de la formación Bucaramanga. Según Julivert (1963) [21] la falla Suárez tuvo activación simultánea o posterior a la depositación del Cuaternario. Al occidente de Girón se presentan depósitos cuaternarios fuertemente basculados. Ingeominas (1997) [19], clasifica la falla Suárez como activa en el sector norte y como potencialmente activa al sur de Girón. La geometría de la falla es la siguiente: longitud acumulada $145 \mathrm{~km}$, azimut $0^{\circ}-20^{\circ}$, el sentido del movimiento es inverso siniestro [18].

\section{- Falla Arrugas}

Se desprende de la Falla La Salina y se extiende hacia el norte. A lo largo de su plano, el bloque oriental constituido por rocas del Cretáceo se ha levantado y está cabalgando rocas terciarias lo- calmente. Pertenece al sistema de fallas del Magdalena. La geometría de la falla es la siguiente: longitud de $64 \mathrm{~km}$, azimut $0^{\circ}-20^{\circ}$, el sentido del movimiento es inverso.

\section{- Falla La Salina}

Esta estructura se localiza al occidente del departamento de Santander, constituyéndose en el límite entre la Secuencia Terciaria del Valle Medio del Magdalena y las rocas Preterciarias de la Cordillera Oriental. Rocas del Cretáceo Superior se encuentran cabalgando rocas Terciarias, se calculan desplazamientos verticales del orden de 1200 m. Algunos sismos se han localizado por la Red Sismológica Nacional de Colombia en el área de la Falla La Salina. Page (1986) [4], menciona que el Sistema de la Falla La Salina es activo, mientras que Ingeominas (1997) [19] considera la falla como potencialmente activa. Según Ingeominas (1988) [19], en algunos sectores la Falla La Salina presenta un movimiento dextral importante. La geometría de la falla es la siguiente: longitud $95 \mathrm{~km}$, azimut $10^{\circ}-30^{\circ}$, el sentido del movimiento es inverso.

\section{- Falla Nus}

Las rocas están principalmente en el bloque occidental, la cual eleva una probable superficie de Erosión del Mioceno cuyos materiales remanentes se caracterizan por estar alineados en bancas estrechas y planas. Se encuentra paralela a la falla Bagre. Pasa por los ríos Honda y Tigre. Tiene una longitud de $80 \mathrm{~km}$, el sentido del movimiento es inverso siniestro lateral.

\section{- Falla Infantes}

Se encuentra al occidente de la falla Arrugas y es la falla más cercana a la ciudad de Barrancabermeja. Atraviesa las quebradas Las Lajas y Vizcaína. Tiene una longitud de $46 \mathrm{~km}$, y su movimiento es siniestro (lateral izquierdo) [9]. 


\section{con-ciencias}

\section{- Nido de Bucaramanga}

Es una de las zonas de mayor actividad sísmica de Colombia. Se origina en la convergencia de la placa del Caribe en dirección E-SE con respecto a la de Sudamérica, lo cual implica un acortamiento paralelo a la dirección de convergencia. Este acortamiento probablemente refleje la subducción de la placa del Caribe bajo el continente. Esta porción de placa subducida, denominada "segmento de Bucaramanga", está limitada al sur por el segmento del Cauca asociado a la subducción de Nazca y contiene al "Nido de Bucaramanga" de sismos de profundidad intermedia. Se registró un sismo fuerte con magnitud Ms de 6.1, ocurrido el 29 de julio de 1967, el cual semi-destruyó el municipio de Betulia. El temblor sacudió la ciudad de Barrancabermeja e incluso produjo pequeños daños en edificaciones [9].

\section{RESULTADOS}

Para labores de diseño y revisión estructural, comúnmente se utilizan los períodos de retorno de 475,1000 y 2000 años. El periodo de retorno de 475 años corresponde a una vida útil de la estructura de 50 años y a una probabilidad de excedencia del 10\%, dicho periodo de retorno es el que se utiliza en las normas de diseño y construcción sismorresistente de estructuras convencionales de vivienda y oficinas. La Fig. 2 presenta las curvas de amenaza sísmica para la ciudad de Barrancabermeja.

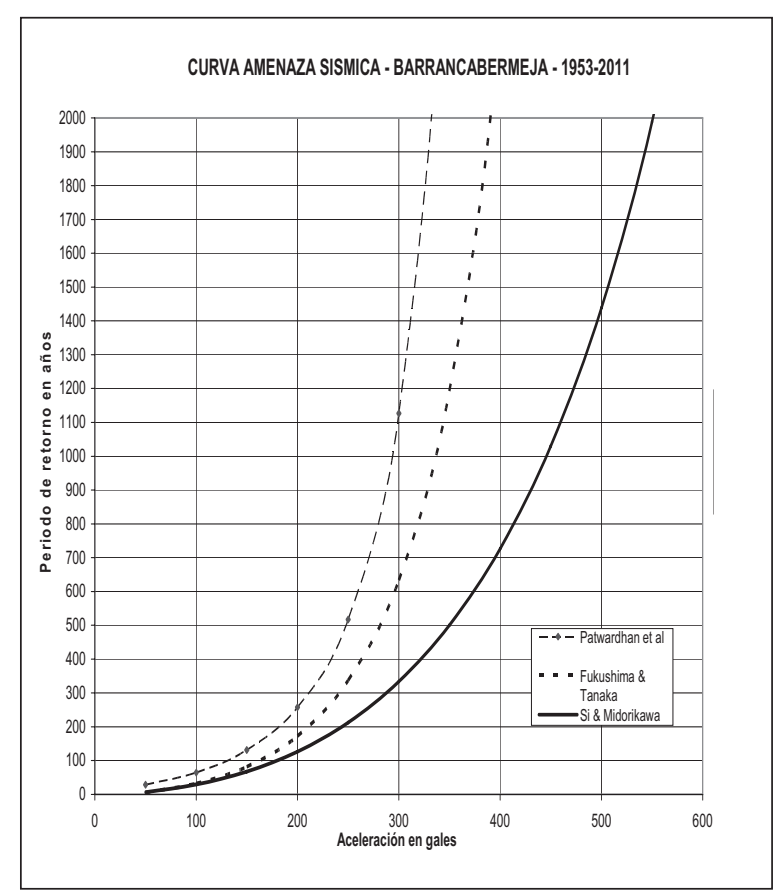

Fig. 2. Curvas de amenaza sísmica para Barrancabermeja.

En la tabla 1 se resumen las aceleraciones pico del terreno para periodos de retorno de 475, 1000 y 2000 años.

Desde el punto de vista determinístico, luego de estimar el valor de Mw para cada falla y la distancia más cercana desde el centro de Barrancabermeja a la falla, se aplican nuevamente las ecuaciones de atenuación y se halla el valor de la aceleración máxima que puede producir dicho sismo. La tabla 2 presenta un ejemplo de los resultados obtenidos [11].

Tabla 1. Aceleraciones pico del terreno para varios periodos de retorno.

\begin{tabular}{|c|c|c|c|c|}
\hline \multirow{2}{*}{$\begin{array}{c}\text { Periodo de retorno } \\
\text { TR en años }\end{array}$} & \multicolumn{2}{|c|}{ Aceleración en gales para periodo de 56 años } & \multirow{2}{*}{$\begin{array}{c}\text { Aceleración } \\
\text { en gales según } \\
\text { NSR-10 }\end{array}$} \\
\cline { 2 - 5 } & $\begin{array}{c}\text { Atenuación Fukushima } \\
\text { \& Tanaka (1990) }\end{array}$ & $\begin{array}{c}\text { Atenuación Patward- } \\
\text { han, et ál (1978) }\end{array}$ & $\begin{array}{c}\text { Si \& Mido- } \\
\text { rikawa (1999) }\end{array}$ & 147 \\
\hline 475 & 280 & 268 & 345 & - \\
\hline 1000 & 330 & 314 & 440 & - \\
\hline 2000 & 393 & 358 & 550 & - \\
\hline
\end{tabular}


Tabla 2. Valores de Mw y aceleración para las diferentes fallas geológicas que podrían afectar Barrancabermeja [11], utilizando la ecuación de Fukushima y Tanaka (1990) [19].

\begin{tabular}{|c|c|c|c|c|c|c|}
\hline Falla & $\begin{array}{c}\text { Distancia a } \\
\text { Barrancaber- } \\
\text { meja (km) }\end{array}$ & $\begin{array}{l}\text { Longitud } \\
\text { (km) }\end{array}$ & $\begin{array}{l}\text { Longitud } \\
\text { estimada } \\
\text { de ruptura } \\
(\mathbf{k m})\end{array}$ & $\begin{array}{l}\text { Magnitud } \\
(\mathrm{Mw})\end{array}$ & $\begin{array}{c}\text { Aceleración en } \\
\text { gales (Ecuacio- } \\
\text { nes atenuación } \\
\text { Fukushima y } \\
\text { Tanaka, 1990) }\end{array}$ & $\begin{array}{l}\text { Aceleración } \\
\text { \%g (Ecuación } \\
\text { Fukushima y } \\
\text { Tanaka, 1990) }\end{array}$ \\
\hline Bagre & 76 & 159.3 & 15 & 6.8 & 71.6 & $7 \% \mathrm{~g}$ \\
\hline Nus & 83 & 80.0 & 15 & 6.8 & 63.2 & $6 \% \mathrm{~g}$ \\
\hline Otú & 86 & 144.4 & 15 & 6.8 & 60.0 & $6 \% \mathrm{~g}$ \\
\hline Palestina & 71 & 430.6 & 30 & 7.1 & 93.2 & $10 \% g$ \\
\hline Cimitarra & 63 & 136.7 & 15 & 6.8 & 91.7 & $9 \% g$ \\
\hline Infantes & 16 & 46.0 & 15 & 6.8 & 305.6 & $31 \% g$ \\
\hline Arrugas & 30 & 64.0 & 15 & 6.8 & 197.2 & $20 \% g$ \\
\hline La Salina & 38 & 95.0 & 15 & 6.8 & 159.7 & $16 \% g$ \\
\hline El Carmen & 55 & 62.0 & 15 & 6.8 & 108.1 & $11 \% g$ \\
\hline Bucaramanga - Santa Marta & 95 & 537.6 & 30 & 7.1 & 61.8 & $6 \% \mathrm{~g}$ \\
\hline Suárez & 78 & 145.0 & 15 & 6.8 & 69.1 & $7 \% g$ \\
\hline
\end{tabular}

Nota: La distancia a Barrancabermeja $(\mathrm{km})$, esta medida desde el centro de la ciudad hasta el punto más cercano de la falla.

\section{DISCUSIÓN Y CONCLUSIONES}

Investigaciones recientes muestran la necesidad de revisar y actualizar los estudios de amenaza sísmica en Colombia [23 - 27] y [11], debido a que los valores obtenidos, con métodos robustos y clásicos, como Hanks y Cornell (1994) [28] presentan valores mayores que la norma vigente.

En el año 2010 ocurrió un sismo catastrófico en Haití que recordó la importancia de tener en cuenta la evidencia histórica (Alfaro y Van Hissenhoven, 2010) [25], por otra parte el sismo de Tohoku - Japón (2011) sobrepasó las expectativas conservadoras de los japoneses (ERI, 2011) [1].

Colombia tiene un faltante importante de información instrumental (Alfaro, 2011) [27] que hace que haya partes del territorio en las cuales no se puede dar información confiable de amenaza sísmica desde el punto de vista probabilístico.
En esta investigación se ha utilizado la ecuación de atenuación de Si \& Midorikawa $(1999,2000)$ [15], [16], ya que los valores instrumentales del reciente sismo de Tohoku se ajustan bastante bien a la misma (ERI, 2011) [1]. En los análisis probabilístico y determinístico los valores obtenidos con dicha ecuación son bastante mayores que los obtenidos con las otras ecuaciones.

La ciudad de Barrancabermeja se encuentra en una zona de amenaza sísmica importante debida a diversas fuentes, entre las cuales se destacan las fallas Cimitarra, Palestina, Bagre, Nus, Arrugas, Salinas, Otú, Infantes y la zona del nido de Bucaramanga.

El análisis probabilístico de la amenaza sísmica para Barrancabermeja, se realizó con datos de 1953 a 2011 utilizando las ecuaciones de atenuación de Patwardhan, et ál. (19789 [13]; Fukushima \& Tanaka (1990) [14] y Si \& Midorikawa (1999) $[15,16]$, donde se evaluaron aceleraciones 


\section{con-ciencias}

pico del terreno para una ventana de tiempo de 56 años que van de 268 a 345 gales para un periodo de retorno de 475 años; 314 a 440 gales para un período de retorno de 1000 años y 358 a 550 gales para un periodo de retorno de 2000 años. La Norma Sismo Resistente NSR-10 establece una aceleración pico en roca para la ciudad de Barrancabermeja con un valor de 0.15 , dicho valor equivale a 147 gales para un período de retorno de 475 años.

Al comparar el promedio de las aceleraciones halladas por medio de las ecuaciones de atenuación [13-15] éstas se encuentran por encima del valor establecido en la normativa colombiana actual (NSR-10), sobrepasando el valor de la norma en un $190 \%, 182 \%$ y $235 \%$ respectivamente.

El sismo máximo creíble para la ciudad de Barrancabermeja, podría producirse por la falla Infantes con una magnitud estimada $\mathrm{Mw}$ de $6.8 \mathrm{y}$ una aceleración 515 gales o $53 \% \mathrm{~g}$, si se aplica la Ecuación de atenuación de Si y Midorikawa
$(1999,2000)[15,16]$. Este valor se podría considerar el techo y de alguna manera controlaría el análisis probabilístico.

El sismo de Quetame - Colombia (24/05/08) con magnitud MS 5.8 generó aceleraciones de 605 gales en la componente NS, 460 gales EW y 297 gales UD en roca a 9 kilómetros del epicentro (Ingeominas, 2008) [29]. Este sismo generó pánico en la zona epicentral, víctimas mortales y cuantiosos daños materiales, incluida toda una serie de movimientos de remoción en masa en la carretera Bogotá - Villavicencio que implicó el cierre de la vía durante varios días. Las aparentemente altas aceleraciones que se presentan en este artículo son coherentes con los registros del último movimiento fuerte del terreno ocurrido en Colombia.

\section{AGRADECIMIENTOS}

El software utilizado en el desarrollo de la Fig. 1 es GMT (Wessel y Smith, 2004) [30]. Este proyecto fue financiado por CIEES.

\section{REFERENCIAS}

[1] The University of Tokio. Earthquake Research Institute, University of Tokyo, 2011. [En línea]. Disponible en: http://outreach. eri.u-tokyo.ac.jp/eqvolc/201103_tohoku/eng.

[2] Ministerio de Minas y Petróleos, Geología del Cuadrángulo H.11, Barrancabermeja. 1966.

[3] J. Goberna, Investigaciones geofisicas sobre las estructuras océano continentales del Occidente colombiano. Bogotá: Instituto Geofísico Universidad Javeriana, 1981.

[4] W. Page, Geología sísmica y sismicidad del Noroeste de Colombia, Bogotá: Interconexión Eléctrica, 1986
[5] H. Flórez, Zonificación sísmica preliminar de Barrancabermeja. Bogotá: Universidad de los Andes, 1993.

[6] AIS (Asociación Colombiana de Ingeniería Sísmica), Ingeominas (Instituto de Investigaciones en Geociencias, Minería y Química) y Universidad de los Andes, Estudio General de Amenaza Sísmica de Colombia. Publicación Especial de Ingeominas. Bogotá. 1996.

[7] A. Alfaro, et ál., Microzonificación sísmica preliminar de Barrancabermeja, Instituto Geofísico Universidad Javeriana y Consultoría Colombiana, Bogotá. 2000. 
[8] Instituto Geofísico Universidad Javeriana y Consultoría Colombiana, Microzonificación sismica preliminar de Barrancabermeja. Memoria Técnica. Bogotá. 2000.

[9] C. González, Incremento de los riesgos naturales en Barrancabermeja debido al aumento de las vulnerabilidades sociales por el desplazamiento interno en Colombia, Trabajo de Grado, Pontificia Universidad Javeriana, Bogotá, 2001.

[10] C. González y A. Alfaro, "El desplazamiento interno detonante de la vulnerabilidad ante amenazas naturales en Barrancabermeja Colombia”. presentado en el Congreso Iberoamericano de Ingeniería Sísmica, Madrid, 2001.

[11] M. Correa, Barrancabermeja: evaluación de la amenaza sísmica de la región. Trabajo de Grado. Facultad Tecnológica. Universidad Distrital Francisco José de Caldas. Bogotá. 2011.

[12] U.S. Geological Survey, Catálogos de sismos ocurridos SISRA y PDE, 2010. [En línea]. Disponible en: http://www.usgs.gov/.

[13] K. Patwardhan, I. Sadigh, Idriss and R. Youngs, Attenuation of Strong Motion Effect of Site Conditions. Transmission Path Characteristics and Focal Depths, submitted to the Bull, Seismological Society of America. 1978.

[14] Y. Fukushima and T. Tanaka, "A new attenuation relation for peak horizontal acceleration of strong earthquake ground motion in Japan," Bulletin of the Seismological Society of America, vol. 80, no. 4, pp. 757-783, Aug. 1990.

[15] H. Si and S. Midorikawa, "Attenuation relations for peak ground acceleration and velocity considering effects of fault type and site condition," Journal of Struct. Construct. Eng. no. 523, pp. 63-70, Japanese, 1999.

[16] H. Si and S. Midorikawa, "New Attenuation Relations for Peak Ground Acceleration and Velocity Considering Effects of Fault Type and Site Condition," Proceedings of 12th World Conference on Earthquake Engineering, 2000.

[17] D. Wells and K. Coppersmith. "New empirical relationships among magnitude, rupture length, rupture width, rupture area, and surface displacement," Seismological Society of America, vol. 84, no. 4, pp. 9741002, Aug. 1994.

[18] G. Paris, M. Machette, R. Dart, and K. Haller, Map and Database of Quaternary Faults and Folds in Colombia and its Offshore Regions, Science for a changing world, USGS 2000.

[19] N. Montes y A. Sandoval, Base de datos de fallas activas de Colombia. Recopilación bibliográfica. Bogotá: Ingeominas. 2001.

[20] M. Julivert, "Observaciones sobre el Cuaternario de la Sabana de Bogotá," Universidad Industrial de Santander, no. 7, pp. 5-36. 1961.

[21] M. Julivert, "Los rasgos tectónicos de la región de la Sabana de Bogotá y los mecanismos de formación de las estructuras," Boletín de Geología Bucaramanga UIS, pp. 13-14, 5-102. 1963.

[22] M. Julivert, "Cover and basement tectonics in the cordillera Oriental of Colombia, South America, and a comparison with some other folded chains," Geological Society of America Bulletin, vol. 81, no. 12, pp. 3623-3646, Dec. 1970. 


\section{con-ciencias}

[23] M. Bohórquez y A. Alfaro, "Análisis probabilístico de la amenaza símica de NeivaColombia - 40 años del macrosismo del Huila (1967-2007)," Revista Épsilon, no. 11, pp. 41-48. July-Dec. 2008.

[24] M. Romero y A. Alfaro, "Dificultades en la evaluación de la amenaza sísmica de Ibagué debido a la escasez de datos". Revista Épsilon, no. 13, pp. 241-253, July-Dec. 2009.

[25] P. Molina y A. Alfaro, "Evaluación de la acción sísmica para Tunja - Colombia," Revista Ciencia e Ingeniería Neogranadina. Bogotá: vol.19, no.2, pp. 17-28, 2009.

[26] A. Alfaro y R. Van Hissenhoven, "Sismo de Haití: aspectos sismológicos," Revista Epsilon, no. 14, pp. 113-120, Jan.-Jun. 2010.

[27] A. Alfaro, "Dificultades en los estudios probabilísticos de amenaza debido a la escasez de datos instrumentales," Sometido a la
Revista Ciencia e Ingeniería Neogranadina. 2011.

[28] Hanks y Cornell, Probabilistic Seismic Hazard Analysis: A Beginner's Guide. In Proceedings of the. Fifth Symposium on Current Issues Related to Nuclear Power Plant Structures, Equipment and Piping, North Carolina State University, Raleigh. pp. I/1-1 a I/1-17. 1994.

[29] Ministerio de Minas y Energía, «El sismo de Quetame (Cundinamarca) del 24 de mayo de 2008. Aspectos sismológicos,» Ingeominas. [En línea]. Disponible en: http://seisan.ingeominas.gov.co/RSNC/ pdfs/Info_quetame.pdf

[30] P. Wessel, y W. Smith, The Generic Mapping Tools Version 4 - Technical Reference and Cookbook, Hawaii: University of Hawaii at manoa. 2006 [En línea]. Disponible en: http://gmt.soest.hawaii.edu 